\title{
Holonomy of Homogeneous Vector Bundles and Polar Representations
}

\section{Carlos Olmos \& Marcos Salvai}

\begin{abstract}
Given a homogeneous vector bundle with a connection, we are concerned with the computation of its holonomy group using the homogeneous structure. In the case of the normal bundle of an irreducible homogeneous submanifold of euclidean space, we obtain that the Lie algebra of the normal holonomy group is algebraically generated by the projection to the normal space at a point of the corresponding Killing fields of the ambient space.
\end{abstract}

1. Introduction. Let $E^{n+k} \stackrel{\pi}{\rightarrow} M^{n}=G / H$ be a homogeneous (real) vector bundle (i.e., $G$ acts on $E$ by bundle morphisms and this action projects down to the $G$-action on $M)$. Let us consider a $G$-invariant connection $\nabla$ on $E \stackrel{\pi}{\rightarrow}$ $M$. A natural question is whether the holonomy group $\Phi_{p}$ of $\nabla$ at a given point $p \in M$ (or its Lie algebra) can be calculated from $G$. The answer is yes for the tangent bundle $T M$ of a homogeneous (locally) irreducible Riemannian manifold $M$ (endowed with the Levi-Civita connection). In fact, Kostant $[\mathrm{K}]$ proved, if $M$ is not Ricci flat, that the Lie algebra of $\Phi_{p}$ is (algebraically) generated by the skew-symmetric endomorphisms of $T_{p} M$ of the form $(\nabla X)_{p}$, where $X$ is an arbitrary Killing field induced by $G$. Twenty years later, Alekseevsky and Kimel'fel'd $[\mathrm{AK}]$ proved that a homogeneous non-flat Riemannian manifold can not be Ricci flat (see also [B, pp. 553]). So, the Lie algebra of the holonomy group of an irreducible homogeneous Riemannian manifold can be computed always by the method of Kostant.

One of the goals of this article is to show that for the normal bundle $\nu(M)$ of an (extrinsic) homogeneous submanifold $M^{n}$ of $\mathbb{R}^{n+k}$ (endowed with the normal connection $\nabla^{\perp}$ ), the normal holonomy group can also be derived, in a nice and geometric way, from $G$. 
Theorem A. Let $G$ be a subgroup of the group of isometries $I\left(\mathbb{R}^{n+k}\right)$ of $\mathbb{R}^{n+k}$ and let $M^{n}=G \cdot v\left(v \in \mathbb{R}^{n+k}, n \geq 2\right)$ be an orbit of such a subgroup which is irreducible and full as a submanifold of $\mathbb{R}^{n+k}$. Then, the Lie algebra of the normal holonomy group $\Phi_{p}$ at $p \in M$ is (algebraically) generated by the (orthogonal) projection, to the affine subspace $p+\nu(M)_{p}$, of the Killing fields on $\mathbb{R}^{n+k}$ (restricted to this subspace) induced by $G$. Moreover, $\Phi_{p}=\bar{G}_{p} \cdot \Phi_{p}^{*}$, where $\bar{G}_{p}=\left\{g_{\mid E_{p}}: g \in G_{p}\right\}, G_{p}$ is the isotropy subgroup of $G$ at $p$ and $\Phi_{p}^{*}$ is the connected component of the identity of $\Phi_{p}$.

If the orbit $M$, in the theorem above, is a principal one for the $G$-action, then the Lie algebra $\mathfrak{k}$, generated by projection of Killing fields to a given normal space, may be interpreted as a measure of how far is the $G$-action on $\mathbb{R}^{n+k}$ from being polar (in which case this Lie algebra would be trivial from the definition of polarity). Observe that $\mathfrak{k}$ depends only on the geometry of $M$ and not on the particular group which has it as an orbit.

We next give the main ideas in the proof of Theorem A. First of all, we prove a general result about the holonomy of arbitrary homogeneous vector bundles. In the notation of the beginning of this section, let us identify any element $X$ in the Lie algebra of $G$ with the field on $E$ defined by $q \rightarrow d /\left.d t\right|_{0} \exp (t X) \cdot q$

Theorem B. Let $E \stackrel{\pi}{\rightarrow} M=G / H$ be a (real) homogeneous vector bundle and let $\nabla$ be a $G$-invariant connection in this fiber bundle. Let $p \in M$ and let $K$ be the connected subgroup of the linear group $G \ell\left(E_{p}\right)$ of the fiber $E_{p}$ whose Lie algebra $\mathfrak{k}$ is generated by the vertical component of fields in $E$ (restricted to $E_{p}$ ) induced by arbitrary elements in the Lie algebra of $G$. Then

(i) $\bar{G}_{p} \cdot K$ is a subgroup of $G \ell\left(E_{p}\right)$ which contains the holonomy group $\Phi_{p}$ and which is contained in the normalizer of this group in $G \ell\left(E_{p}\right)$ (resp. in $O\left(E_{p},\langle,\rangle_{p}\right)$, if the connection and the $G$-action preserve a pseudometric $\langle$,$\rangle on the fibers), where \bar{G}_{p}=\left\{g_{\mid E_{p}}: g \in G_{p}\right\}$.

(ii) The Lie algeba $\mathfrak{k}$ contains the Lie algebra of $\Phi_{p}$ and is contained in the normalizer of this Lie algebra in $\mathfrak{g l}\left(E_{p}\right)$ (resp. in $\mathfrak{s o}\left(E_{p},\langle,\rangle_{p}\right)$, if the connection and the $G$-action preserve a pseudometric $\langle$,$\rangle on the fibers).$

The proof of Theorem A follows from the result above and the fact that the connected component of the normalizer (in the full orthogonal group) of a connected group acting as an $s$-representation (i.e., the isotropy representation of a semisimple symmetric space) coincides with itself. Recall that the nontrivial part of the normal holonomy group is always an $s$-representation [O1]. For the trivial factor of the normal holonomy we have to use the results in [O2].

We next give an application of Theorem A. For a given orbit $M^{n}=G \cdot v$, $v \neq 0$ of a compact connected Lie subgroup $G$ of $S O(n+k)$, let $J_{v}$ be the 
intersection of the affine normal spaces $w+\nu(G \cdot w)_{w}$ where $w$ runs over the elements of the affine normal space $v+\nu(M)_{v}$. (Any affine normal space, in our context, contains the origin and hence it may be replaced by a linear subspace). It is easy to check that $v$ belongs always to $J_{v}$ and consequently $\operatorname{dim}\left(J_{v}\right) \geq 1$. If $G$ acts polarly on $\mathbb{R}^{n+k}$ and $M^{n}$ is a principal orbit, then $\operatorname{dim}\left(J_{v}\right)=k$. Assume now that $M^{n}$ is irreducible and full as a submanifold of $\mathbb{R}^{n+k}$ and $n \geq 2$ (e.g., if $G$ acts irreducibly and the ambient space has dimension at least 3 ). We will show in this case, using Theorem $\mathrm{A}$, that $\operatorname{dim}\left(J_{v}\right) \leq \operatorname{rank}(M)$, where $\operatorname{rank}\left(M^{n}\right)$ is the maximal number of linearly independent (locally defined) parallel normal vector fields to $M$ (in fact, it can be shown the equality). By applying the main result in [O2] we obtain the following generalization of the Theorem of J. Dadok $[\mathrm{D}]$.

Theorem C. Let $G$ be a compact connected Lie subgroup of $S O(n+k)$ and let $M^{n}=G \cdot v, v \in \mathbb{R}^{n+k}, n \geq 2$, be an orbit of $G$ which is irreducible and full as a submanifold of $\mathbb{R}^{n+k}$. Let $J_{v}$ be the intersection of the affine normal spaces $w+\nu(G \cdot w)_{w}$ where $w$ runs over the elements of the affine normal space $v+\nu\left(M^{n}\right)_{v}$. Assume that $\operatorname{dim}\left(J_{v}\right) \geq 2$. Then there exists a Lie subgroup $\tilde{G}$ of $S O(n+k)$ containing $G$, acting as an s-representation and such that $M^{n}$ is also an orbit of $\tilde{G}$

2. Holonomy of homogeneous vector bundles. Let $E^{n+k} \stackrel{\pi}{\rightarrow} M^{n}$ be a (real) vector bundle over a connected manifold $M^{n}$. Let $\nabla$ be a linear connection on $E$, and let $\mathcal{K}: T E \rightarrow E$ denote the connection operator associated to $\nabla$, that is, $\mathcal{K}(\xi)=D v / d t(0)$, where $v(t)$ is a curve in $E$ with $\dot{v}(0)=\xi$ and $D v / d t$ is the covariant derivative along the curve $c=\pi \circ v$. Let us suppose, additionally, that there is a left action of a connected Lie group $G$ on $E$ which preserves both the vector bundle structure of $E$ and the connection $\nabla$. The group action on $E$ projects down to a left action on $M$, which we assume to be transitive (we will denote both actions by a dot).

We fix $p \in M$ for the remainder of the paper. Let $\Phi_{p}$ be the holonomy group of $\nabla$ at $p$ and let $L\left(\Phi_{p}\right)$ denote its Lie algebra.

Given $X \in \mathcal{G}$, the Lie algebra of $G$, and $v \in E$, we denote $\xi_{X}(v):=X \cdot v=$ $d /\left.d t\right|_{0} \exp (t X) \cdot v$. Let $A_{X}$ be the endomorphism of $E_{p}$ defined by

$$
A_{X}(v)=\mathcal{K}\left(\xi_{X}(v)\right)=\mathcal{K}(X \cdot v) .
$$

We have that $A_{X}$ is linear, since it is of class $C^{1}$ and $A_{X}\left(a v_{0}\right)=a A_{X}\left(v_{0}\right)$ holds for each $a \in \mathbb{R}$ and $v_{0} \in E_{p}$. In fact, we have that

$$
a A_{X}\left(v_{0}\right)=a \mathcal{K}\left(\xi_{X}\left(v_{0}\right)\right)=\left.a \frac{D}{d t}\right|_{0} \exp (t X) \cdot v_{0}=\left.\frac{D}{d t}\right|_{0} \exp (t X) \cdot a v_{0}=A_{X}\left(a v_{0}\right)
$$


Let $\mathfrak{k}$ be the smallest Lie subalgebra of $\operatorname{End}\left(E_{p}\right)$ which contains all the $A_{X}$, with $X \in \mathcal{G}$, and let $K$ be the connected subgroup of $G \ell\left(E_{p}\right)$ whose Lie algebra is k. Let $G_{p}$ be the isotropy subgroup of $G$ at $p$ and let

$$
\bar{G}_{p}=\left\{g_{\mid E_{p}}: g \in G_{p}\right\} .
$$

Proposition 2.1. We keep the general notation and assumptions of this section. Then

(i) $\bar{G}_{p} K$ is a subgroup of $G \ell\left(E_{p}\right)$ which contains the holonomy group $\Phi_{p}$.

(ii) The Lie algebra of $\Phi_{p}$ is contained in $\mathfrak{k}$.

Proof. (i) We first show that $\bar{G}_{p} K$ is a group. It suffices to show that $g K g^{-1}=K$ for all $g \in \bar{G}_{p}$, or equivalently, since $K$ is connected, that $\operatorname{Ad}(g) \mathfrak{k}=\mathfrak{k}$ for all $g \in \bar{G}_{p}$, where Ad is the adjoint action of $G \ell\left(E_{p}\right)$ on its Lie algebra. This is true since $\operatorname{Ad}(g)$ leaves invariant the generator set of $\mathfrak{k}$. Indeed, one can easily verify that $\operatorname{Ad}(g) A_{X}=g \circ A_{X} \circ g^{-1}=A_{\operatorname{Ad}(g) X}$ for all $X \in \mathcal{G}$. This shows that $\bar{G}_{p} K$ is a subgroup of $G \ell\left(E_{p}\right)$.

We will need to consider the principal bundle $B \stackrel{\tilde{\pi}}{\rightarrow} M$ of frames of $E$, that is, $B=\left\{b: \mathbb{R}^{k} \rightarrow E_{q} \mid b\right.$ is a linear isomorphism, $\left.q \in M\right\}$. We consider in $B \stackrel{\tilde{\pi}}{\rightarrow} M$ the connection $\mathcal{H}$ associated to $\nabla$ (the action of $G$ on $B$ is the natural action induced by the action on $E$ ).

We fix $b_{0} \in B_{p}=\tilde{\pi}^{-1}(p)$ and consider the holonomy subbundle $Q\left(b_{0}\right)$ through $b_{0}$, that is, $Q\left(b_{0}\right)=\{b(1) \mid b:[0,1] \rightarrow B$ is a horizontal piecewise differentiable curve with $\left.b(0)=b_{0}\right\}$. The subset $Q\left(b_{0}\right)$ is a principal subbundle of $B$ with structure group isomorphic to $\Phi_{p}$.

We define $P=G \cdot K \cdot b_{0}=\left\{g f b_{0} \mid g \in G, f \in K\right\}$. We will show that $P$ is a principal subbundle of $B$ with structure group $\bar{G}_{p} K$. There must exist a subgroup $\tilde{H}$ of $G \ell\left(\mathbb{R}^{k}\right)$ such that $P_{p}:=\tilde{\pi}^{-1}(p) \cap P=G_{p} \cdot K \cdot b_{0}=b_{0} \tilde{H}$ (using the first assertion that we have proved). Then, $P \cdot \tilde{H}=\left(G \cdot K \cdot b_{0}\right) \cdot \tilde{H}=G$. $\left(G_{p} \cdot K \cdot b_{0}\right) \cdot \tilde{H}=G \cdot b_{0} \cdot \tilde{H} \cdot \tilde{H}=G \cdot b_{0} \cdot \tilde{H}=P$. It is now standard to show that $P$ is a principal subbundle of $B$ with structure group $\tilde{H}$.

We next prove that $Q\left(b_{0}\right) \subset P$. It suffices to show that $\mathcal{H}_{b} \subset T_{b} P$ for all $b \in P$, where $\mathcal{H}$ is the horizontal distribution. Since $G$ leaves $P$ invariant and preserves the connection, it suffices only to show (using the right invariance of $\mathcal{H}$ ) that $\mathcal{H}_{b_{0}} \subset T_{b_{0}} P$. If $\eta \in \mathcal{H}_{b_{0}}$ is arbitrary, since $G$ acts transitively on $M$, there exists $X \in \mathcal{G}$ such that $d \tilde{\pi}(\eta)=X \cdot p$. Define $\zeta=d /\left.d t\right|_{0} \exp (t X) \cdot b_{0}=X \cdot b_{0} \in$ $T_{b_{0}} P$ (observe that $\left.d \tilde{\pi}(\zeta)=d \tilde{\pi}(\eta)=X \cdot p\right)$. Let $\psi=\zeta-d /\left.d t\right|_{0} \exp \left(t A_{X}\right) \cdot b_{0} \in$ $T_{b_{0}} P$ (it belongs to $T_{b_{0}} P$ because $A_{X}$ belongs to the Lie algebra $\mathfrak{k}$ of $K$ ). We have that $d \tilde{\pi}(\psi)=d \tilde{\pi}(\eta)=X \cdot p$, because $d /\left.d t\right|_{0} \exp \left(t A_{X}\right) \cdot b_{0}$ is vertical. If we 
prove that $\psi$ is horizontal, then it must coincide with $\eta$. This would prove that $\eta$ belongs to $T_{b_{0}} P$. For it, let

$$
\psi_{i}=X \cdot\left(b_{0}\left(e_{i}\right)\right)-\left.\frac{d}{d t}\right|_{0} \exp \left(t A_{X}\right) \cdot\left(b_{0}\left(e_{i}\right)\right)
$$

where $e_{1}, \ldots, e_{k}$ is the canonical basis of $\mathbb{R}^{k}$. Then $\mathcal{K}\left(\psi_{i}\right)=\mathcal{K}\left(X \cdot\left(b_{0}\left(e_{i}\right)\right)\right)-$ $A_{X}\left(b_{0}\left(e_{i}\right)\right)=\mathcal{K}\left(\xi_{X}\left(b_{0}\left(e_{i}\right)\right)\right)-A_{X}\left(b_{0}\left(e_{i}\right)\right)=0$ by the definition of $A_{X}$. Hence $\eta$ is horizontal.

(ii) Let $D \in L\left(\bar{G}_{p}\right)$, the Lie algebra of $\bar{G}_{p}$. There exists $X \in \mathcal{G}$ such that $D(v)=d /\left.d t\right|_{0} \exp (t X) \cdot v$ for all $v \in E_{p}$. Since $\exp (t X) \cdot v \in E_{p}$ for all $t$, we have that $D(v)=\xi_{X}(v)=A_{X}(v)$. Consequently, $L\left(\bar{G}_{p}\right) \subset \mathfrak{k}$. Thus the connected component of the identity of $\bar{G}_{p}$ is contained in $K$. Thus, $L\left(\bar{G}_{p} K\right)=L(K)=\mathfrak{k}$ and (ii) follows now from (i).

In the case that $G$ preserves a given holonomy subbundle (and hence any) of the principal bundle $B$ of frames of $E$, we have not only the inclusion but the equality in Proposition 2.1. More precisely,

Lemma 2.2. We keep the general notation and assumptions of this section. Assume, furthermore, that for any $g \in G$ there exists a piecewise differentiable curve $c:[0,1] \rightarrow M$ with $c(0)=p=\tilde{\pi}\left(b_{0}\right), c(1)=g \cdot p$ and such that the parallel transport $\tau_{c}$ along $c$ coincides with $g_{\mid E_{p}}$. Then

(i) The Lie algebra of $\Phi_{p}$ coincides with $\mathfrak{k}$.

(ii) $\bar{G}_{p} K=\Phi_{p}$.

Proof. By Proposition 2.1, it suffices to show that $P \subset Q\left(b_{0}\right)$. Since $P$ is $G$-invariant and $Q\left(b_{0}\right)$ is so by assumptions, we need only to show that $K \cdot b_{0} \subset$ $Q\left(b_{0}\right)$. If $X \in \mathcal{G}$ and $v \in E_{p}$ we have that

$$
A_{X}(v)=\mathcal{K}\left(\xi_{X}(v)\right)=\left.\frac{D}{d t}\right|_{0} \exp (t X) \cdot v=\left.\frac{d}{d t}\right|_{0}\left(\tau_{t}\right)^{-1}(\exp (t X) \cdot v)
$$

where $\tau_{t}$ denotes the parallel transport along the curve $s \mapsto \exp (s X) \cdot p$ restricted to $[0, t]$. Hence, from the assumptions, the smooth curve $t \mapsto\left(\tau_{t}\right)^{-1} \circ \exp (t X)$ in $G \ell\left(E_{p}\right)$ is actually contained in the holonomy group $\Phi_{p}$ and consequently, by $(*)$, $A_{X}$ belongs to the Lie algebra $L\left(\Phi_{p}\right)$. Thus, $K \subset \Phi_{p}$ and therefore $K \cdot b_{0} \subset P$. 
Remark 2.3. We keep the notation of this section and, in particular, that of the lemma above. Then the smooth curve $t \mapsto\left(\tau_{t}\right)^{-1} \circ \exp (t X)$ is always contained in the normalizer, in $G \ell\left(E_{p}\right)$, of the holonomy group $\Phi_{p}$. Moreover it is not difficult to see that it defines a 1-parameter subgroup (using that $g \circ \tau_{c} \circ$ $g^{-1}=\tau_{g \circ c}$, for any curve $c$ in $M, g \in G$ ). So, one obtains the following general formula for the parallel transport along the curve $t \mapsto \exp (t X) \cdot p$

$$
\tau_{t}=\exp (t X) \circ e^{-t A_{X}}
$$

3. The pseudoriemannian case. In this section we assume, additionally, that $(M, g)$ is pseudoriemannian, that each fibre $E_{q}$ of $E$ carries a non degenerate bilinear form $\langle,\rangle_{q}$ which depends smoothly on $q$, and that the connection is metric, i.e., $v\langle U, V\rangle=\left\langle\nabla_{v} U, V\right\rangle+\left\langle U, \nabla_{v} V\right\rangle$ for each vector field $v$ on $M$ and $U, V$ sections of $E$. Under these conditions $\Phi_{q}$ is contained in $O\left(E_{q},\langle,\rangle_{q}\right)=$ \{orthogonal maps of $E_{q}$ with respect to $\langle,\rangle_{q}$ \} and thus its Lie algebra $L\left(\Phi_{q}\right)$ is contained in $\mathfrak{s o}\left(E_{q},\langle,\rangle_{q}\right)=$ sskew-symmetric maps on $E_{q}$ with respect to $\left.\langle,\rangle_{q}\right\}$, for all $q \in M$.

We consider on $E$ the canonical pseudoriemannian metric (called the Sasaki metric), i.e., the map

$$
\left.d \pi\right|_{v} \times\left.\mathcal{K}\right|_{T_{v} E}: T_{v} E \rightarrow T_{q} M \times E_{q}
$$

is a lineal pseudoisometry for each $v \in E_{q}, q \in M$ (the bilinear form in $T_{q} M \times E_{q}$ is $\left.g_{q} \times\langle,\rangle_{q}\right)$. We furthermore require that the action of $G$ on $M$ is by isometries (with respect to $g$ ) and that the action on $E$ maps isometrically (with respect to $\langle\rangle$,$) fibers into fibers. Then, since G$ preserves $\nabla, G$ acts on $E$ by isometries with respect to the Sasaki metric.

In this context, $A_{X}$ is a skew-symmetric endomorphism of $E_{q}$ with respect to $\langle,\rangle_{q}$ (or equivalently a Killing field on $E_{q}$ which vanishes at the origin), since for $v \in E_{q}$ we have

$$
\begin{aligned}
\left\langle A_{X} v, v\right\rangle & =\left\langle\mathcal{K}\left(\xi_{X}(v)\right), v\right\rangle=\left\langle\left.\frac{D}{d t}\right|_{0} \exp (t X) \cdot v, v\right\rangle \\
& =\left.\frac{1}{2} \frac{d}{d t}\right|_{0}\langle\exp (t X) \cdot v, \exp (t X) \cdot v\rangle=\left.\frac{1}{2} \frac{d}{d t}\right|_{0}\langle v, v\rangle=0
\end{aligned}
$$

This follows also from the following interpretation: the field $\xi_{X}$ is a Killing field on $E$ and hence the orthogonal projection of $\xi_{X \mid E_{q}}$ to $T E_{q}$ is Killing in $E_{q}$, since the fibers are totally geodesic in $E$ (see [Be, Prop. 1.102]). 
Lemma 3.1. Let $p \in M$ and let $g_{t}$ be a smooth curve in $G$ with $g_{0}=e$. Let $c$ be the curve in $M$ defined by $c(t)=g_{t} \cdot p$. Let $\tau_{t}: E_{p} \rightarrow E_{c(t)}$ denote the parallel transport along $c$. Then

(i) $\left(\tau_{t}\right)^{-1} g_{t}$ lies in the identity component of the normalizer $N\left(\Phi_{p}\right)$, in $O\left(E_{p},\langle,\rangle_{p}\right)$, of $\Phi_{p}$, for all $t$.

(ii) The endomorphism $A_{X}$ of $E_{p}$ is contained in the normalizer, in $\mathfrak{s o}\left(E_{p},\langle,\rangle_{p}\right)$, of $L\left(\Phi_{p}\right)$.

Proof. (i) Given $h \in \Phi_{p}$, there exists a piecewise smooth loop $\gamma:[0,1] \rightarrow M$ at $p$ such that $h$ is the parallel transport along $\gamma$. Now,

$$
\left(g_{t}^{-1} \tau_{t}\right)^{-1} h\left(g_{t}^{-1} \tau_{t}\right)=\left(\tau_{t}\right)^{-1} g_{t} h g_{t}^{-1} \tau_{t} \in \Phi_{p}
$$

since one can verify that it is the parallel transport along the curve obtained by juxtaposition of $\left.c\right|_{[0, t]}, g_{t} \cdot \gamma$ and the reverse of $\left.c\right|_{[0, t]}\left(g_{t}\right.$ preserves the connection). Finally, the curve $t \rightarrow\left(\tau_{t}\right)^{-1} g_{t}$ is contained in the identity component of $N\left(\Phi_{p}\right)$ since it is continuous.

Using the formula $(*)$ in the proof of Lemma 2.2 and part (i), we easily obtain (ii) by choosing $g_{t}=\exp (t X), X \in \mathcal{G}$.

Corollary 3.2. If the Lie algebra of $\Phi_{p}$ coincides with its own normalizer in $\mathfrak{s o}\left(E_{p},\langle,\rangle_{p}\right)$, then it coincides with k. Moreover, $\Phi_{p}=\bar{G}_{p} K$.

Remark 3.3. If the holonomy group $\Phi_{p}$ acts on $E_{p}$ as the isotropy representation of a semisimple Riemannian symmetric space, then the Lie algebra of the holonomy group coincides with its own normalizer in the Lie algebra of the full orthogonal group (this is indeed well known, see e.g., [O2, Lemma 5.2]). This is indeed the case of the nontrivial part of the normal holonomy group representation of a submanifold of a space of constant curvature [O1]. This is also the case of the holonomy group representation of an irreducible Riemannian manifold of non-zero scalar curvature (see $[\mathrm{S}]$ ). Applying Corollary 3.2 to this last case we obtain part (b) of Theorem 4.5 of Kostant $[\mathrm{K}]$, under the stronger assumption of non-zero scalar curvature instead of non-vanishing Ricci tensor.

Proof of Theorem B. It follows immediately from Proposition 2.1 and Lemma 3.1

Proof of Theorem A. From [O2, Theorem C] (see [O3, Theorem 1.1] for the non compact case) we obtain that the hypothesis of Lemma 2.2 are fulfilled and hence its assertion is true.

We are now concerned with the proof of Theorem C. We need the following result. 
Lemma 3.4. Under the assumptions and notation of Theorem $C$, then $\operatorname{dim}\left(J_{v}\right) \leq \operatorname{rank}\left(M^{n}\right)$.

Proof. Let $w \in v+\nu\left(M^{n}\right)_{v}=\nu\left(M^{n}\right)_{v}$. We have that $J_{v}$ is a linear subspace because it passes through the origin. Then $w+J_{v}$ is contained in $\nu(G \cdot w)_{w}$ because both $\{w\}$ and $J_{v}$ are contained in this normal space. Then, if $X$ belongs to the Lie algebra $\mathfrak{g}$ of $G, X \cdot w$ is perpendicular to $J_{v}$. Hence the projection to $\nu\left(M^{n}\right)_{v}$ of Killing fields of $\mathbb{R}^{n+k}$ (restricted to this subspace), induced by elements of $\mathfrak{g}$, lie in the foliation $w \mapsto w+\left(J_{v}\right)^{\perp}$ of $\nu\left(M^{n}\right)_{v}$. Hence any iterated bracket of projections of those Killing fields to $\nu\left(M^{n}\right)_{v}$ belongs to this foliation. Then, applying Theorem A, $Z \cdot w$ is perpendicular to $J_{v}$, if $Z$ belongs to the Lie algebra of the normal holonomy group $\Phi_{v}$ of $M^{n}$. Then $J_{v}$ must be contained in the set of fixed points of the connected component $\Phi_{v}^{*}$ of the normal holonomy group. Since $\operatorname{rank}\left(M^{n}\right)$ is the dimension of the subspace of $\nu\left(M^{n}\right)_{v}$ which consists of all fixed points of $\Phi_{v}^{*}$, we obtain the lemma.

Proof of Theorem C. Lemma 3.4 and [O2, Thm. A] imply that $M^{n}$ is also the orbit of a subgroup $\tilde{G}$ of $S O(n+k)$ which acts as an $s$-representation. Since $\operatorname{rank}\left(M^{n}\right) \geq 2, \tilde{G}$ does not act transitively on the unit sphere of $\mathbb{R}^{n+k}$. Hence, using the results in $[S], \tilde{G}$ must contain $G$.

Remark. Any s-representation, except eleven possible exceptions, arises as the normal holonomy representation of a (homogeneous) submanifold of Euclidean space (see $[\mathrm{HO}]$ ). It is not known if some of these exceptions do really occur as normal holonomy. Theorem A could be helpful to produce new normal holonomy groups.

Acknowledgment. The authors would like to thank Isabel Dotti for her useful comments about Ricci flat homogeneous manifolds.

\section{REFERENCES}

[AK] D. V. Alekseevsky \& B. N. Kimel'FeL'D, Structure of homogeneous riemannian spaces with zero Ricci curvature, Functional Anal. Appl. 9 (1975), 97-102.

[B] L. BÉRARD-BERGERY, Sur la courbure des métriques riemanniennes invariantes des groupes de Lie et des espaces homogénes, Ann. Sci. École Norm. Sup. 4 (1978), 543-576.

[Be] A. BESSE, Manifolds all of whose geodesic are closed, series Ergebnisse der Mathematik und ihre Grenzgebiete 93, Berlin-Heidelberg-New York: Springer (1978).

[D] J. DADOK, Polar coordinates induced by actions of compact Lie groups, Trans. Amer. Math. Soc. 288 (1985), 125-137.

[HO] E. Heintze $\&$ C. Olmos, Normal holonomy groups and s-representations, Indiana Univ. Math. J. 41 No. 3 (1992), 869-874.

[K] B. Kostant, Holonomy and the Lie algebra of infinitesimal motions of a Riemannian manifold, Trans. Amer. Math. Soc. 80 (1955), 528-542. 
[O1] C. Olmos, The normal holonomy group, Proc. Amer. Math. Soc. 110 (1990), 813-818.

[O2] - Homogeneous submanifolds of higher rank and parallel mean curvature, J. Differential Geometry 39 (1994), 605-627.

[O3] _ Orbits of rank one and parallel mean curvature, Trans. Amer. Math. Soc. $\mathbf{3 4 7}$ (1995), 2927-2939.

[S] J. Simons, On the transitivity of holonomy systems, Ann. of Math. 76 (1962), 213-234.

The authors were supported in part by the Universidad Nacional de Córdaba, CONICET and CONICOR.

Fa.M.A.F.

Universidad Nacional de Córdoba

Ciudad Universitaria

5000 Córdoba, Argentina

E-MAIL: olmos@mate.uncor.edu

E-MAIL: salvai@mate.uncor.edu

Received: March 20th, 1995. 